\title{
Galectin-1 knockdown improves drug sensitivity of breast cancer by reducing P-glycoprotein expression through inhibiting the Raf-1/AP-1 signaling pathway
}

\author{
Fang Wang ${ }^{1, *}$, Pengwei Lv ${ }^{1, *}$, Yuanting Gu${ }^{1}$, Lin Li ${ }^{1}$, Xin $\mathbf{G e}^{1}$, Guangcheng Guo ${ }^{1}$ \\ ${ }^{1}$ Department of Breast Surgery, The First Affiliated Hospital of Zhengzhou University, 450000, China \\ *These authors have contributed equally to this work \\ Correspondence to: Lin Li, email: huangjinlingv@sina.com \\ Keywords: Galectin-1, multidrug resistance, P-glycoprotein, Raf-1/AP-1, breast cancer \\ Received: October 19, $2016 \quad$ Accepted: January 16, $2017 \quad$ Published: February 15, 2017 \\ Copyright: Wang et al. This is an open-access article distributed under the terms of the Creative Commons Attribution License \\ (CC-BY), which permits unrestricted use, distribution, and reproduction in any medium, provided the original author and source \\ are credited.
}

\section{ABSTRACT}

Galectin-1 (Gal-1), a member of the galectin family of carbohydrate binding proteins, plays a pivotal role in various cellular processes of tumorigenesis. The regulatory effect of Gal-1 on multidrug resistance (MDR) breast cancer cells is still unclear. QRT-PCR and western blot showed that Gal-1 and MDR gene 1 (MDR1) were both highly expressed in breast tumor tissues and cell lines. MTT assay and flow cytometry revealed that Gal-1 knockdown improved sensitivity to paclitaxel (PTX) and adriamycin (ADR) in MCF-7/PTX and MCF-7/ADR cells via inhibition of cell viability and promotion of cell apoptosis, while MDR1 overexpression weakened the sensitivity to PTX and ADR induced by Gal-1 knockdown. Furthermore, the negative effects of Gal-1 knockdown on sensitivity to PTX and ADR in MCF-7/PTX and MCF-7/ADR cells were revealed to be mediated via the suppression of Raf-1/AP-1 pathway. In conclusion, Gal-1 knockdown dramatically improved drug sensitivity of breast cancer by reducing P-glycoprotein (P-gp) expression via inhibiting the Raf-1/AP-1 pathway, providing a novel therapeutic target to overcome MDR in breast cancer.

\section{INTRODUCTION}

Breast cancer is one of the most prevalent cancers and the gradually increasing incidence of breast cancer is the leading cause of cancer death among women in the world [1]. Chemotherapy is the main treatment of breast cancer. Chemotherapeutic agents such as paclitaxel (PTX) and adriamycin (ADR) have been widely used in the treatment of various solid tumors including breast cancer [2]. However, multidrug resistance (MDR) gradually emerges in clinical trials by using chemotherapeutic agents alone or combined with other antineoplastic agents and is becoming a major clinical obstacle and paramount factor in the failure of breast cancer chemotherapy and the human body has become insensitive to antitumor drugs due to MDR [3].

The mechanisms related to MDR are multifarious, such as the increased drug efflux, activation of DNA repair process and the deregulation of drug-induced apoptosis and the major factor of MDR pathogenesis is the high expression of MDR proteins of adenosine triphosphate (ATP)-binding cassette (ABC) transporters in cell membranes, especially multidrug efflux transporter P-glycoprotein (P-gp) [4, 5]. P-gp, a plasma membrane glycoprotein encoded by the ATP-binding cassette subfamily $\mathrm{B}$ member 1 (ABCB1, also known as multidrug resistance gene 1 (MDR1)), is one of the main multidrug transporters and has an important impact on the absorption, distribution and elimination of chemotherapeutic drugs [6]. P-gp can recognize chemotherapeutic drugs as substrates, pump substrates out of tumor cells and restrict the uptake of substrates in an ATP-dependent manner [7], which leads to a decrease of intracellular drug accumulation and soon causes resistance to numerous drugs in cancer cells [8]. Therefore, P-gp is regarded as the most important MDRregulatory drug target and inhibition of P-gp expression would be an effective therapeutic approach to overcome MDR and enhance the therapeutic effect. 
Galectin-1 (Gal-1), a homodimer of 14 kDa subunits, is a member of the galectin family of carbohydrate binding proteins and contains two $\beta$-galactoside binding sites [9]. Gal-1 plays a pivotal role in various cellular processes of tumorigenesis, such as cell proliferation, apoptosis, cell invasiveness, migration, and angiogenesis and regulates the interaction between tumor cells and components of tumor microenvironment [10]. Besides, Gal-1 has been demonstrated as a therapeutic target by identifying the overexpression of Gal-1 in human cancer including breast cancer $[10,11]$.

Raf, a member of Raf family of serine/threonine kinases, is ubiquitously expressed and activated by the Ras family of GTPases, which is involved in survival, cell growth and differentiation of tumors [12]. Raf is involved in a broad range of cancers and functions as an oncogene that regulates mitogen-activated protein (MAPK) pathway and cell proliferation to promote tumourigenesis [13]. The activation of MAPK pathway, which is regulated by phosphorylation of some signaling protein kinases including Ras, Raf, and Raf upstream activator PKC, has important roles in mediating signals caused by growth factors, cytokines, and environment stress and is associated with cell proliferation, survival, differentiation and apoptosis [14]. It has been reported that an intact Gal-1 interface of stacked dimers of H-Ras, Raf and Gal-1 as building blocks is required for Gal-1 to modulate Ras nanoclustering, thus representing a potential drug target site in cancer associated Ras-mutations [15]. The protooncogenes c-Jun and c-Fos are the main components of the AP-1 transcription factor complex in the downstream of Ras/ Raf and are crucial for Ras/Raf-mediated cell proliferation and transformation [16]. Additionally, AP-1 regulates the activation of numerous genes transcription and is a main target of MAPK signaling pathway [17]. AP-1 is associated with various cellular processes, such as cell differentiation, proliferation, survival and apoptosis [18].

In this study, we aimed to investigate the effect of Gal-1 knockdown on cell proliferation and apoptosis of the MDR breast cancer cell lines and the relationship between Gal-1 and Raf-1/AP-1 signaling pathway to further study the molecular mechanism of MDR, providing a novel therapeutic approach to overcome MDR in breast cancer.

\section{RESULTS}

\section{Gal-1 and MDR1 levels were both upregulated in breast tumor tissues and cell lines}

To evaluate the potential role of Gal-1 and MDR1 in breast cancer, the expression levels of Gal-1 and MDR1 in MCF-10A, MCF-7, MCF-7/PTX, MCF-7/ADR cells, normal tissues and breast tumor tissues samples were detected by qRT-CPR and western blot. As shown in Figure 1A and 1B, qRT-PCR results indicated that Gal and MDR1 were significantly upregulated in breast tumor tissues compared with that in normal tissues. In addition, the mRNA expressions of Gal-1 in breast cancer patients were positively correlated with MDR1 $(r=0.736$, $P=0.0001$ ) (Figure 1C). Besides, Gal-1 was found to be dramatically higher in mRNA and protein levels in MCF-7 cells than that in MCF-10A cells and the mRNA and protein levels of Gal-1 in MCF-7/PTX and MCF-7/ ADR cells were strikingly higher than that in MCF-7 cells (Figure 1D, 1E and 1F). The expressions of MDR1 mRNA and protein P-gp in MCF-7 cells were significantly higher than that in MCF-10A cells and dramatically lower than that in MCF-7/PTX and MCF-7/ADR cells (Figure $1 \mathrm{G}, 1 \mathrm{H}$ and $1 \mathrm{I})$. These differential expressions suggested that Gal-1 and MDR1 may play important roles in breast cancer development and resistance.

\section{Gal-1 knockdown enhanced sensitivity to PTX and ADR in MCF-7/PTX and MCF-7/ADR cells}

To determine cell sensitivity to PTX and ADR, MCF-7, MCF-7/PTX and MCF-7/ADR cells were treated with different concentrations of PTX or ADR for $24 \mathrm{~h}$. Cell survival rates were assessed by MTT assay and the results showed that the cell survival rates of MCF-7/PTX cells at $10 \mathrm{nM}, 15 \mathrm{nM}$ and $20 \mathrm{nM}$ of PTX and MCF-7/ ADR cells at $200 \mathrm{nM}, 300 \mathrm{nM}$ and $400 \mathrm{nM}$ of ADR were significantly higher than those in MCF-7 cells (Figure 2A and 2B), which confirmed the PTX resistance in MCF-7/ PTX cells and ADR resistance in MCF-7/ADR cells. IC50 of MCF cells to PTX (15 nM) and IC50 of MCF cells to ADR (300 nM) were chosen for sequential studies.

Considering the upregulation of Gal-1 in MCF7/PTX and MCF-7/ADR cells, Gal-1 knockdown was performed to observe its effect on the drug resistance of MCF-7/PTX and MCF-7/ADR cells. The cell viability and apoptosis in MCF-7/PTX and MCF-7/ADR cells transfected with Gal-1 siRNA1 or Gal-1 siRNA2 were evaluated by MTT assay and flow cytometry with or without PTX or ADR treatment. As presented in Figure 2C and 2D, Gal-1 siRNA1 and Gal-1 siRNA2 significantly decreased the levels of Gal-1 in MCF-7/ PTX and MCF-7/ADR cells, indicating the efficiency of siRNAs in silencing Gal-1 expression. Gal-1 knockdown or (PTX or ADR) treatment resulted in an obvious decrease in cell viability; however, combination of Gal-1 knockdown with PTX or ADR resulted in a lower cell viability than chemotherapy group alone in MCF-7/ PTX and MCF-7/ADR cells (Figure 2E and 2F). Gal-1 knockdown or (PTX or ADR) treatment led to a marked increase in cell apoptosis; however, combination of Gal1 knockdown with PTX or ADR induced a higher cell apoptosis than chemotherapy group alone in MCF-7/PTX and MCF-7/ADR cells (Figure $2 \mathrm{G}$ and $2 \mathrm{H}$ ). These results showed that Gal-1 knockdown improved the sensitivity against PTX and ADR in MCF-7/PTX and MCF-7/ADR cells via the inhibition of cell viability and induction of cell apoptosis. 


\section{Overexpression of MDR1 reduced the sensitivity to PTX and ADR induced by Gal-1 knockdown in MCF-7/PTX and MCF-7/ADR cells}

To explore the effect of Gal-1 knockdown or MDR1 overexpression on P-gp protein in MCF-7/PTX and MCF7/ADR cells, cells were transfected Gal-1 siRNA1, Gal1 siRNA2, (Gal-1 siRNA1 + pcDNA-MDR1) or (Gal-1 siRNA2 + pcDNA-MDR1). The western blot results indicated that Gal-1 siRNA1 and Gal-1 siRNA2 both significantly decreased the levels of P-gp in MCF-7/PTX and MCF-7/ADR cells compared with corresponding control groups, while MDR1 overexpression alleviated the inhibition effects of Gal-1 siRNA1 or Gal-1 siRNA2 on P-gp (Figure 3A and 3B).
To explore the effect of overexpression of MDR1ondrug sensitivity induced by Gal-1 knockdown in MCF-7/PTX and MCF-7/ADR cells, cells were treated with (Gal-1 siRNA1 + drug) or (Gal-1 siRNA1 + drug + pcDNA-MDR1). The MTT assay showed that combination of Gal-1 siRNA1 and PTX or Gal-1 siRNA1 and ADR strikingly suppressed the relative cell viability of MCF-7/PTX and MCF-7/ADR cells compared to (Con siRNA + drug) group, whereas MDR1 overexpression reversed these negative effects (Figure $3 \mathrm{C}$ and 3D). Flow cytometry assay indicated that Gal-1 siRNA1 and PTX or Gal-1 siRNA1 and ADR obviously induced apoptosis of MCF-7/PTX and MCF-7/ADR cells compared with (Con siRNA + drug) group, while MDR1 overexpression attenuated the induction effects
A

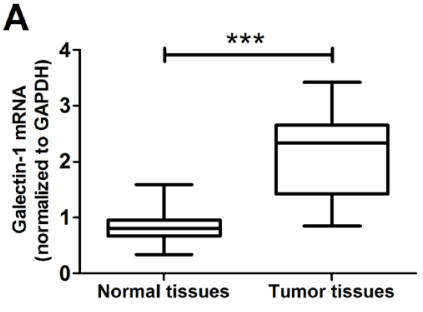

D
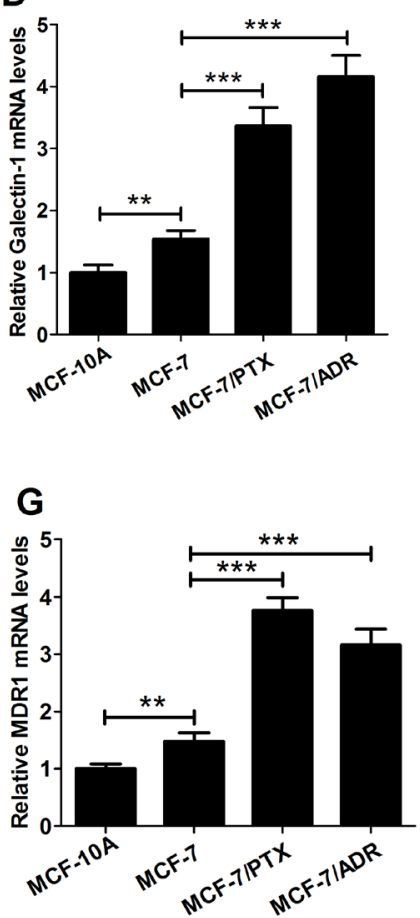

B

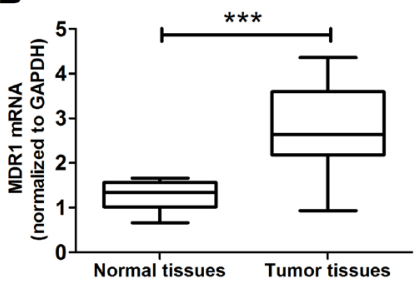

E

Galectin-1

$\beta$-actin

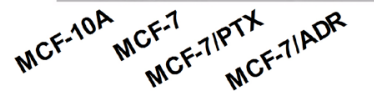

H

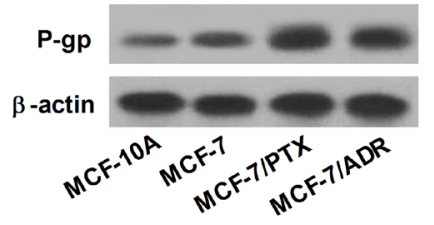

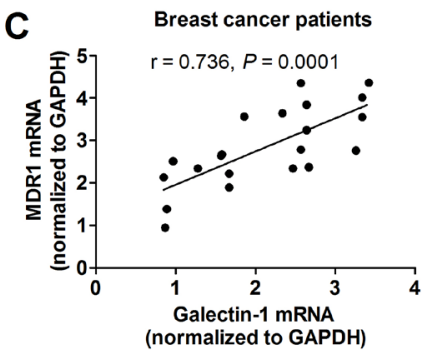

$\mathbf{F}$

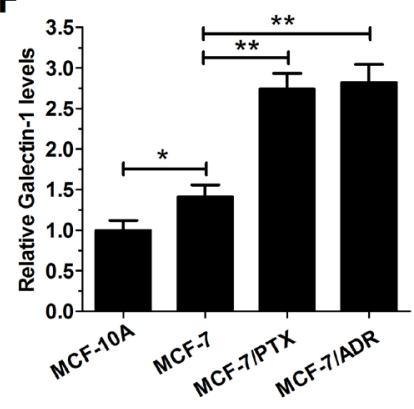

I

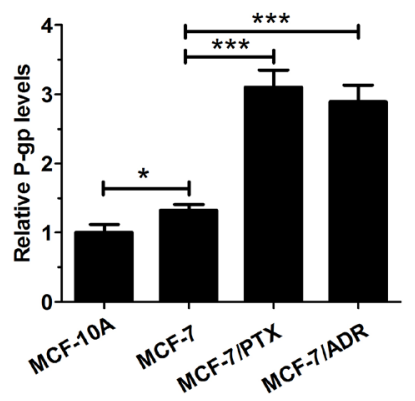

Figure 1: Expression of Gal-1 and MDR1 in breast tumor tissues and cells lines. A. qRT-PCR was used to measure the level of Gal-1 mRNA (A) and MDR1 mRNA B. in breast tumor tissues and normal tissues. C. A positive correlation between MDR1 and Gal-1 mRNA expression. D. The mRNA expressions of Gal-1 in normal breast cell line (MCF-10A) and breast tumor cell lines (MCF-7, MCF-7/ PTX and MCF-7/ADR) cells were detected by qRT-PCR. E. and F. The levels of Gal-1 protein in MCF-10A, MCF-7, MCF-7/PTX and MCF-7/ADR cells were evaluated by western blot. G. The mRNA expressions of MDR1 in MCF-10A, MCF-7, MCF-7/PTX and MCF-7/ ADR cells were determined by qRT-PCR. H. and I. The levels of P-gp protein in MCF-10A, MCF-7, MCF-7/PTX and MCF-7/ADR cells were detected by western blot. Data are shown as mean $\pm \mathrm{SD} . * P<0.05,{ }^{* *} P<0.01$. 

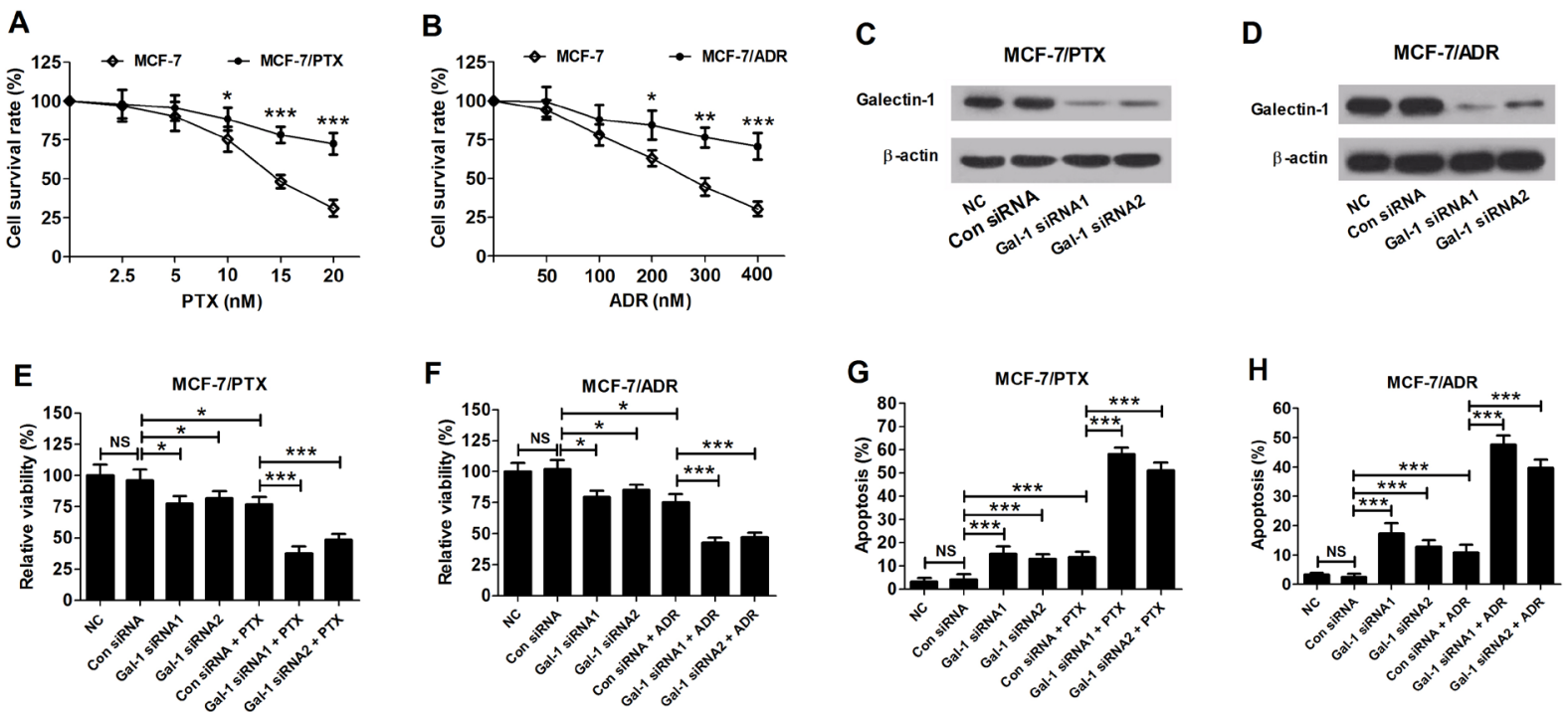

Figure 2: Effects of Gal-1 knockdown on sensitivity to PTX and ADR in MCF-7/PTX and MCF-7/ADR cells. A. Cell survival rates in MCF-7 and MCF-7/PTX with a series of PTX concentrations $(2.5,5,10,15$, and $20 \mathrm{nM})$ treatment were detected by MTT assay. B. Cell survival rates in MCF-7 and MCF-7/ADR with a series of ADR concentrations (50, 100, 200, 300, and 400 nM) treatment were detected by MTT assay. Western blot was used to detect the levels of Gal-1 in MCF-7/PTX cells C. and MCF-7/ADR cells D. transfected with Gal-1 siRNA1, Gal-1 siRNA2 or si-control. E. and G. The relative cell viability and cell apoptosis in MCF-7/PTX cells with Gal-1 siRNA1 or Gal-1 siRNA2 transfection or together with 15 nM PTX treatment was determined by MTT assay and flow cytometry. F. and $\mathbf{H}$. The relative cell viability and cell apoptosis in MCF-7/ADR cells with Gal-1 siRNA1 or Gal-1 siRNA2 transfection or together with $300 \mathrm{nM}$ ADR treatment was determined by MTT assay and flow cytometry. Data are presented as mean $\pm \mathrm{SD} .{ }^{*} P<0.05$, $* * P<0.01, * * * P<0.001$.

A
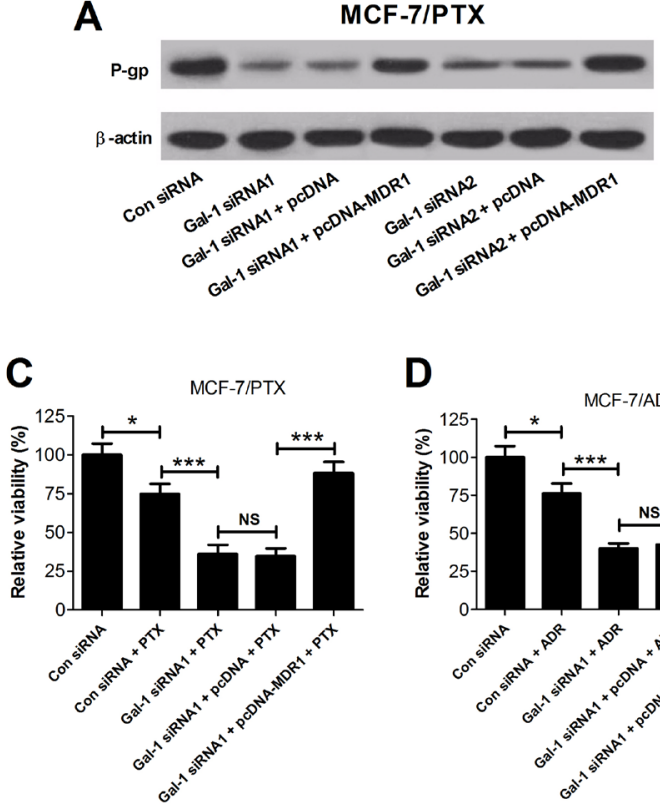

D

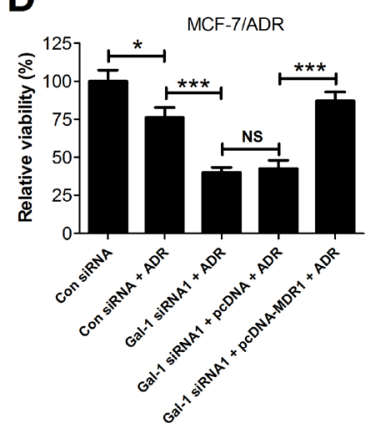

B

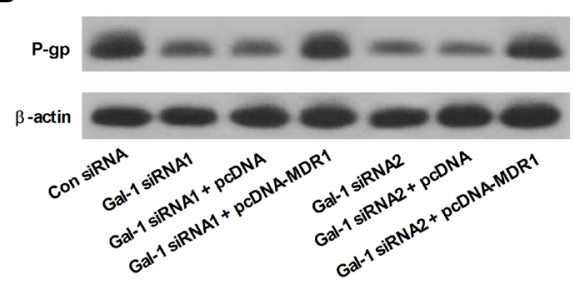

E

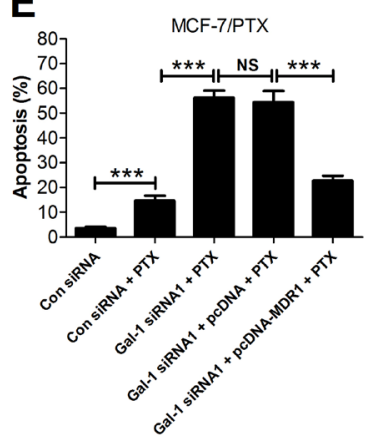

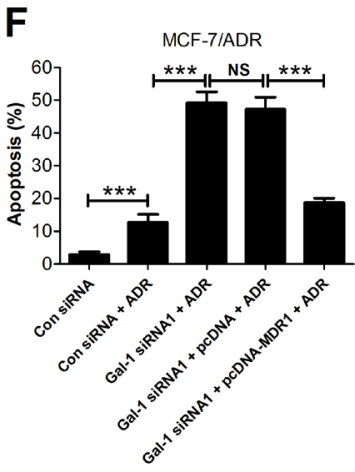

Figure 3: Effects of MDR1 overexpression on the sensitivity to PTX and ADR induced by Gal-1 knockdown in MCF7/PTX and MCF-7/ADR cells. The levels of P-gp in MCF-7/PTX cells A. and MCF-7/ADR cells B. cotransfected Gal-1 siRNAs or (Gal-1 siRNAs + pcDNA-MDR1) were detected by western blot. C. and E. With $15 \mathrm{nM}$ PTX treatment, the relative cell viability and cell apoptosis in MCF-7/PTX cells cotransfected Gal-1 siRNA1 with pcDNA 3.1-MDR1 were evaluated by MTT assay and flow cytometry. D. and F. With $300 \mathrm{nM}$ ADR treatment, the relative cell viability and cell apoptosis in MCF-7/ADR cells cotransfected Gal-1 siRNA1 with pcDNA 3.1-MDR1 were evaluated by MTT assay and flow cytometry. Data are presented as mean $\pm \mathrm{SD} .{ }^{*} P<0.05,{ }^{* * *} P<0.001$. 
(Figure 3E and 3F). These data demonstrated that overexpression of MDR1 significantly decreased the sentivity to PTX and ADR induced by Gal-1 knockdown in MCF-7/PTX and MCF-7/ADR cells via the increase of cell viability and reduction of cell apoptosis.

\section{Gal-1 knockdown significantly suppressed the Raf-1/AP-1 signaling pathway}

The levels of p-Raf-1 (Ser338), Raf-1, p-c-Jun (Ser73), c-Jun and c-Fos were determined by western blot to investigate the effect of Gal-1 knockdown on the Raf-1/AP-1 signaling pathway. As compared with Con siRNA group, Gal-1 siRNA1 and Gal-1 siRNA2 significantly decreased the levels of p-Raf-1 (Ser338), p-c-Jun (Ser73), c-Jun and c-Fos in MCF-7/PTX and MCF-7/ADR cells, indicating that Gal-1 knockdown dramatically decreased the phosphorylation of Raf- 1 and the induction of AP-1 activity in MCF-7/PTX and MCF-7/ADR cells (Figure 4A and 4B).

\section{Galectin-1 knockdown enhanced sensitivity to PTX and ADR by reducing P-glycoprotein expression through inhibiting the Raf-1/AP-1 signaling pathway}

The Raf-1 siRNAs and Raf-1 inhibitor GW5074 were used to inhibit Raf-1 activity to further observe the underlying mechanism of Gal-1 knockdown on MDR of breast cancer cells. The western blot results showed that Raf-1 siRNA1 and Raf-1 siRNA2 dramatically decreased the levels of P-gp, p-Raf-1 (Ser338), Raf-1, p-c-Jun

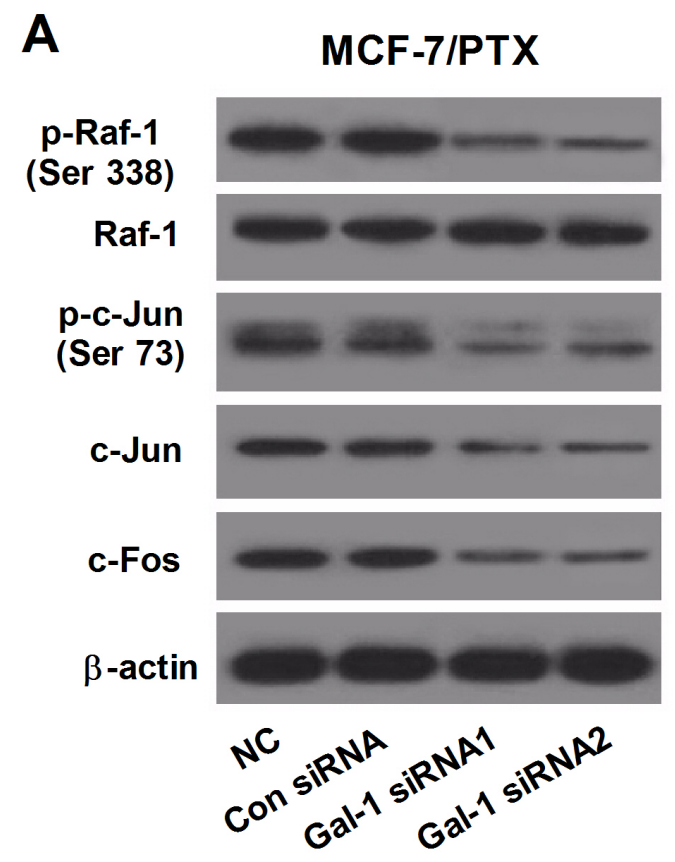

(Ser73), c-Jun and c-Fos in MCF-7/PTX cells (Figure 5A), and Raf-1 inhibitor GW5074 obviously reduced the levels of P-gp, p-Raf-1 (Ser338), p-c-Jun (Ser73), c-Jun and c-Fos in MCF-7/ADR cells (Figure 5B), suggesting the validity of Raf-1 siRNAs and Raf-1 inhibitor GW5074 in suppressing the Raf-1/AP-1 signaling pathway.

The relative cell viability and apoptosis in MCF-7/PTX cells treated with (Raf-1 siRNA1 + PTX) or (Raf-1 siRNA1 + pcDNA-MDR1 + PTX) were assessed by MTT assay and flow cytometry. The results showed that combination of Raf1 siRNA1 and PTX dramatically inhibited cell viability and induced cell apoptosis in MCF-7/PTX cells compared with (PTX + Con siRNA) group, whereas MDR1 overexpression abated these effects (Figure 5C and 5E). Additionally, MCF7/ADR cells were treated with GW5074 or (GW5074 + ADR) or (GW5074 + pcDNA-MDR1 + ADR). MTT assay and flow cytometry showed that combination of GW5074 and ADR significantly decreased cell viability and promoted apoptosis in MCF-7/ADR cells compared with ADR treatment group, while MDR1 overexpression overturned these effects (Figure 5D and 5F). These data suggested that Galectin-1 knockdown enhanced sensitivity aganist PTX and ADR by decreasing P-gp expression through suppressing the Raf-1/AP-1 signaling pathway

\section{DISCUSSION}

Accumulating evidence reveals that Gal-1, a member of the mammalian $\beta$-galactoside-binding proteins, is upregulated in different tumor types, such

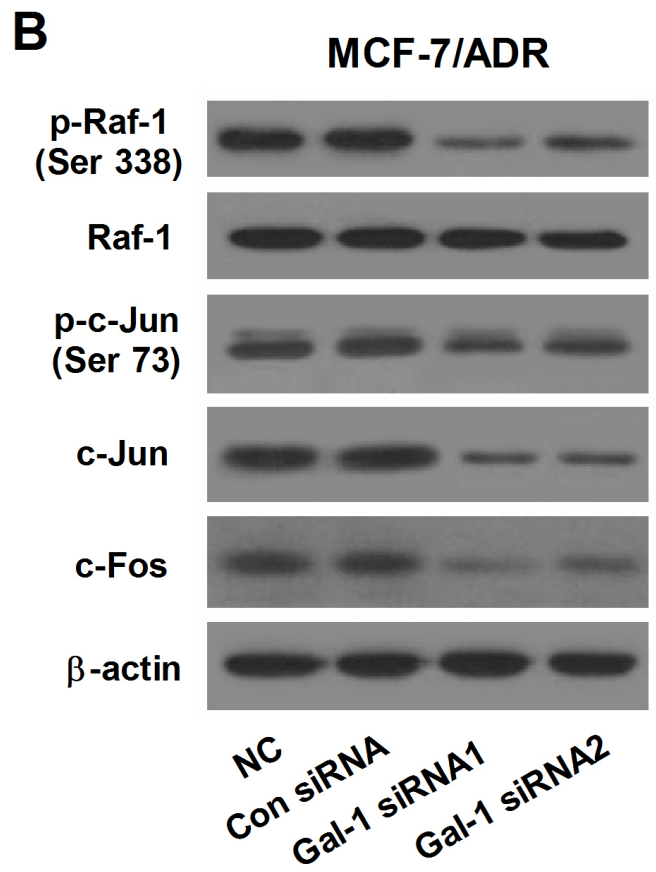

Figure 4: Gal-1 knockdown significantly suppressed the Raf-1/AP-1 signaling pathway. The levels of p-Raf-1 (Ser338), Raf-1, p-c-Jun (Ser73), c-Jun and c-Fos in MCF-7/PTX cells A. and MCF-7/ADR cells B. transfected with Gal-1 siRNA1, Gal-1 siRNA2 or Con siRNA were determined by western blot $\beta$-actin was used for normalization. 
A

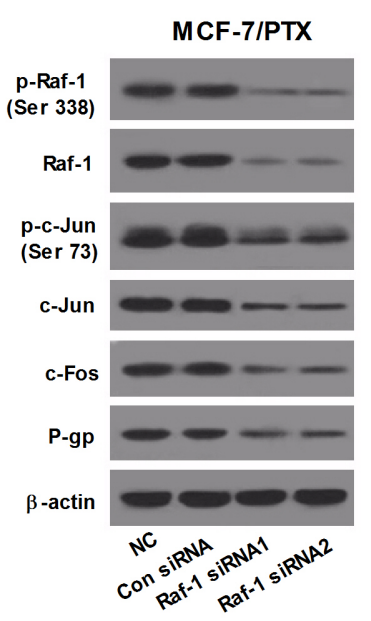

C

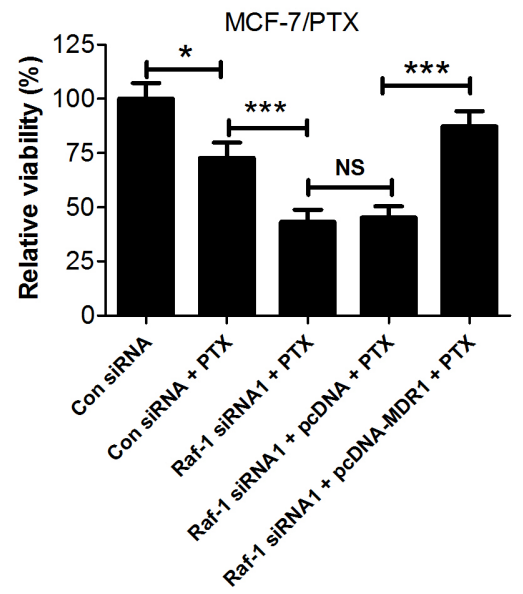

$\mathbf{E}$

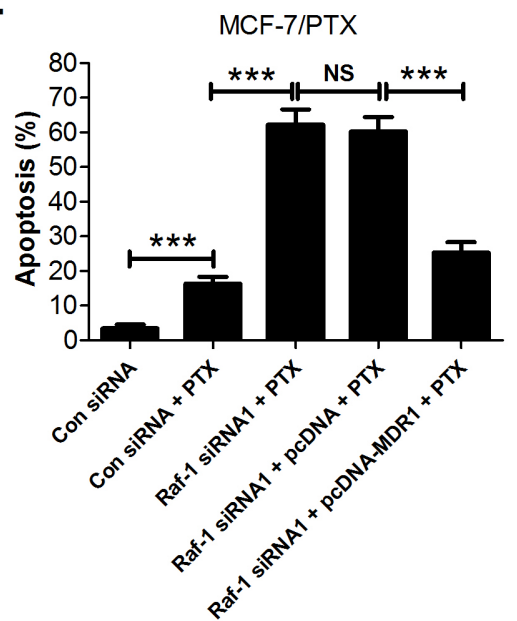

B

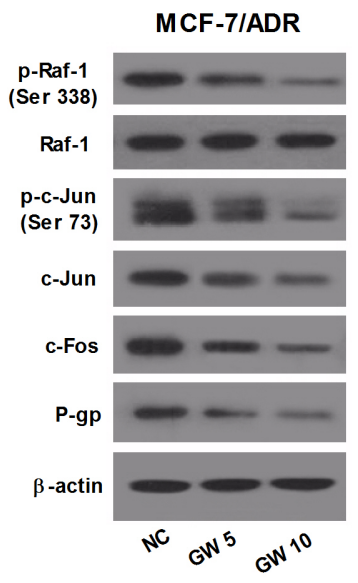

D

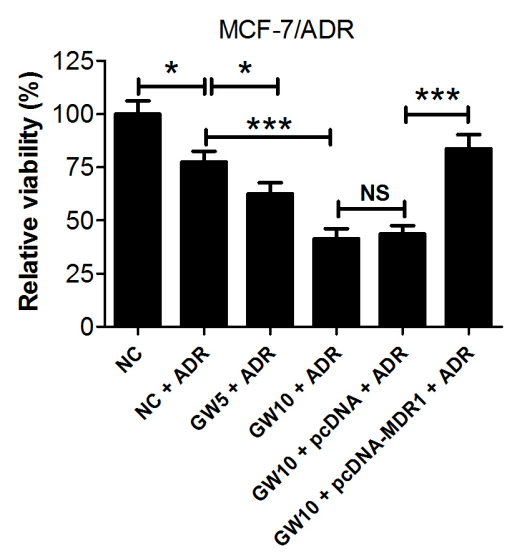

$\mathbf{F}$

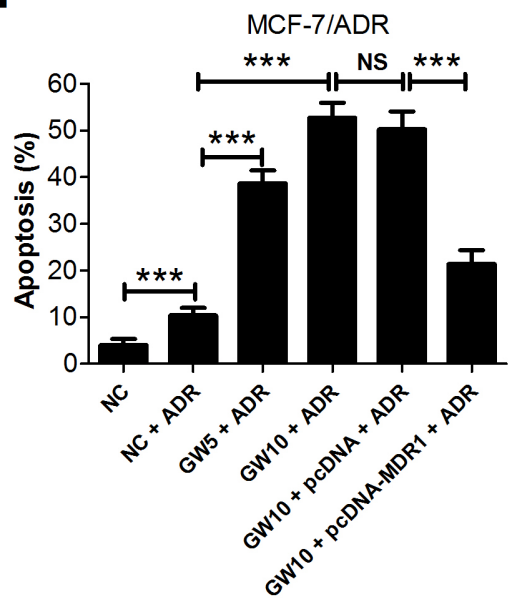

Figure 5: Gal-1 knockdown enhanced sensitivity to PTX and ADR by reducing P-glycoprotein expression through inhibiting the Raf-1/AP-1 signaling pathway. A. The levels of P-gp, p-Raf-1 (Ser338), Raf-1, p-c-Jun (Ser73), c-Jun and c-Fos in MCF-7/PTX cells with Raf-1 siRNA1, Raf-1 siRNA2 or si-control transfection were detected by western blot. $\beta$-actin was used for normalization. B. The levels of P-gp, p-Raf-1 (Ser338), Raf-1, p-c-Jun (Ser73), c-Jun and c-Fos in MCF-7/ADR cells with GW5074 (5 and $10 \mu \mathrm{M}$ ) treatment were detected by western blot. $\beta$-actin was used for normalization. C. and E. With $15 \mathrm{nM} \mathrm{PTX}$ treatment, the relative cell viability and apoptosis in MCF-7/PTX cells cotransfected Raf-1 siRNA1 with pcDNA 3.1-MDR1 were assessed by MTT assay and flow cytometry. D. and F. With $300 \mathrm{nM}$ ADR treatment, the relative cell viability and apoptosis in MCF-7/ADR cells with $10 \mu \mathrm{M}$ GW5074 treatment and pcDNA 3.1-MDR1 transfection were assessed by MTT assay and flow cytometry. Data are presented as mean \pm SD. ${ }^{*} P<$ $0.05, * * * P<0.001$. 
as breast cancer [19], lung cancer [20], and prostate cancer [21]. Gal-1 has distinct biological roles in tumor progression, including cell growth, apoptosis, metastasis, and immunosuppression and is involved in poor prognosis and the metastatic phenotype [22, 23]. For example, Zhang et al. reported that Gal-1 overexpression promoted tumorigenesis and Gal-1 knockdown led to the reduction in cell growth, migration and invasion in epithelial ovarian cancer (EOC) [24]. Zheng et al. showed that overexpression of Gal-1 promoted cell invasion and migration and suppressed cell apoptosis by enhancing TGF- $\beta$ signaling in gastric cancer [25]. Wiest et al. manifested that Gal-1 showed apoptotic potential in human breast cancer and trophoblast tumor cells [26]. Zhu et al. reported that Gal-1 was highly expressed in human breast tumor tissues and Gal-1 knockdown in carcinomaassociated fibroblasts inhibited cell migration and invasion by suppressing the expression of matrix metalloprotein 9 (MMP-9) [19]. In the present study, we demonstrated that Gal-1 was highly expressed in breast tumor tissues and cells as compared with normal tissues and cells. Besides, Gal-1 expression was significantly higher in MDR breast cancer cells MCF-7/PTX and MCF-7/ADR. Gal-1 knockdown dramatically increased sensitivity to ADR and PTX in MCF-7/PTX and MCF-7/ADR cells by inhibiting cell proliferation and enhancing apoptosis.

MDR to chemotherapeutic agents is the main cause of chemotherapy failure of breast cancer. P-pg/MDR1 overexpression is one of the main causes leading to MDR phenomenon in breast cancer and plays a crucial role in producing MDR in breast cancer cells [27]. For example, He et al. reported that MDR1 silencing resulted in a decrease of P-pg activity and drug sensitivity of yolk sac carcinoma [28]. Liu et al. manifested that siMDR1 effectively reduced the expression of MDR 1 and led to a remarkable increase of chemosensitivity to PTX [29]. In the present study, we discovered that the mRNA and protein expression levels of MDR1 were higher in breast tumor tissues and cells than that in normal breast tissues and cells and MDR1 overexpression obviously decreased sensitivity to ADR and PTX induced by Gal-1 knockdown in MCF-7/PTX and MCF-7/ADR cells by promoting cell proliferation and suppressing apoptosis.

Previous studies have reported that Raf and AP-1 signaling pathway is related to MDR [30]. For example, Mukherjee et al. reported that Her2/Raf-1/MAPK/AP-1 pathway contributed to the development of castrateresistance prostate cancer, resulting in early relapse and reduced disease-specific survival [31]. Kreiseder et al. reported that the cytoskeletal linker protein $\alpha$-catulin chemosensitized melanoma cells by activating NF- $\kappa \mathrm{B}$ and AP-1 [32]. Yang et al. found that upregulation of miR195 improved the sensitivity to ADR in breast cancer by suppressing Raf-1 [33]. In addition, it is convincingly demonstrated that both AP-1 and NF- $\mathrm{KB}$ were transcription factors of P-gp [34]. More importantly, NF- $\kappa B$ was demonstrated to induce drug resistance by improving the
MDR1 gene expression in cancer cells [35]. Moreover, a previous study uncovered that ectopic expression of Gal-1 mediated chemoresistence of chronic myeloid leukemia cells by inducing MDR1 expression via P38 MAPK activation and NF- $\kappa B$ translocation [36]. In the present study, we noted that Gal-1 knockdown markedly reduced the levels of P-pg, p-Raf-1 (Ser338), p-c-Jun (Ser 73), c-Jun and c-Fos. These results manifested that Gal-1 knockdown enhanced sensitivity against PTX and ADR by reducing P-glycoprotein expression via suppressing the Raf-1/AP-1 signaling pathway

In conclusion, we found that Gal-1 and P-gp were both upregulated in breast tumor tissues and cells and Gal1 knockdown dramatically improved drug sensitivity of breast cancer cells by reducing P-gp expression through inhibiting the Raf-1/AP-1 signaling pathway, providing a novel evidence that combining the depletion of Gal-1 with drugs PTX or ADR is a potential method for the therapy of patients with breast cancer.

\section{MATERIALS AND METHODS}

\section{Cell lines and tumor tissues}

Breast tumor tissues and corresponding normal tissues from 20 patients with breast cancer were collected from the First Affiliated Hospital of Zhengzhou University. The experiments were carried out with approval of the Ethic Committee of Zhengzhou University. Informed consent was obtained from all patients for using their tissues. The human breast cancer cell line (MCF-7) and human breast epithelial cell line (MCF-10A) were obtained from the American Type Culture Collection (ATCC, Manassas, VA, USA). MCR-7 derived adriamycin (ADR) resistant cells MCF7/ADR and paclitaxel (PTX) resistant cells MCF-7/PTX were established as previously described [37], which were induced drug resistance by pre-treating MCF-7 cells with continuously increasing concentrations of ADR and PTX over 8 months. The MCF-7 and MCF-10A cells were grown in RPMI-1640 medium (Gibco-BRL, Carlsbad, CA, USA) containing 10\% fetal bovine serum (FBS) (Gibco-BRL), 100 $\mathrm{U} / \mathrm{mL}$ penicillin/streptomycin/kanamycin (Genom Co., Ltd., Hangzhou, China) at $37^{\circ} \mathrm{C}$ in a humidified atmosphere of $5 \% \mathrm{CO}_{2}$.

\section{Cell transfection}

Double strand siRNA nucleotides targeting Gal-1 (Gal-1 siRNA1 and Gal-1 siRNA2), double strand siRNA nucleotides targeting Raf-1 (Raf-1 siRNA1 and Raf-1 siRNA2), scrambled siRNA (Con siRNA), pcDNA 3.1MDR1, and pcDNA 3.1 empty vectors were purchased from Shanghai GenePharma Co., Ltd (Shanghai, China). For transient transfection, 50,000 cells were seeded into six-well plates and incubated for $24 \mathrm{~h}$ at $37^{\circ} \mathrm{C}$. Then the cells were transfected with different siRNAs, pcDNAMDR1 or respective controls by Lipofectamine 2000 
(Invitrogen, Grand Island, NY, USA) and incubated for $48 \mathrm{~h}$ in the serum-free medium. The cells with scrambled siRNA transfection were used as the control.

\section{Cell viability analysis}

MCF, MCF-7/ADR, and MCF-7/PTX cells $\left(2 \times 10^{4}\right.$ cells/well) were incubated in 96-well plates for $24 \mathrm{~h}$ and then the cells were transferred into $100 \mu \mathrm{l}$ fresh culture medium containing different concentrations of PTX (2.5, $5,10,15$, and $20 \mathrm{nM})$ or $\operatorname{ADR}(50,100,200,300$, and $400 \mathrm{nM}$ ). Cells with no additives were used as the control. Following $48 \mathrm{~h}$ incubation, $20 \mu \mathrm{l}(0.5 \mathrm{mg} / \mathrm{ml})$ MTT was added to each well and incubated for an additional $4 \mathrm{~h}$ at $37^{\circ} \mathrm{C}$ and then $200 \mu \mathrm{l}$ DMSO was added to dissolve the MTT formazan precipitate for $15 \mathrm{~min}$ after the medium was removed. After the crystals were completely dissolved, the absorbance value at $490 \mathrm{~nm}$ in each well was analyzed by a microplate reader (SPECTRAmax; Molecular Device Corp., Sunnyvale, CA, USA). The IC50 values were calculated using the non-linear regression curve fit of the Graphpad Prism5 software. The cell viability in all transfected cells were detected using the method described above.

\section{Quantitative real-time PCR (qRT-PCR)}

The RAN from the breast cancer cell lines, normal breast tissue and breast tumor tissue samples were isolated by TransZolTMUp (TransGen Biotech, Beijing, China). For the detection of Gal-1 and MDR1 mRNA expression, the complementary DNA (cDNA) was reversely transcribed using RT reagent kit (Takara, Shiga, Japan). The RT-PCR was used to evaluate the mRNA expression of Gal-1 and MDR1 with the SYBR green detection system (ThermoScientific, Waltham, MA, USA) in an ABI7500 sequence detection system (Life Technologies, Carlsbad, CA, USA). The procedure of RTPCR was performed as follows: denaturing temperature $95^{\circ} \mathrm{C}$ for $30 \mathrm{~s}, 60^{\circ} \mathrm{C}$ for $30 \mathrm{sec}, 35$ cycles at $95^{\circ} \mathrm{C}$ for 30 $\mathrm{s}$, and $60^{\circ} \mathrm{C}$ for $20 \mathrm{~s}$. The primers used were as follows: forward, 5'-CTGTGCCTGCACTTCAACC-3' and reverse, 5'-CATCTGGCAGCTTGACGGT-3' for Gal-1; forward, 5'-GGAGCCTACTTGGTGGCACATAA-3' and reverse, 5'-TGGCATAGTCA GGAGCAAATGAAC-3' for MDR1. The mRNA expression of GAPDH was used as the endogenous control.

\section{Western blot}

For western blot analysis, the cells $\left(1 \times 10^{6} / \mathrm{ml}\right)$ with all siRNAs, pcDNA 3.1 MDR1 or respective controls transfection or with GW5074 (5 or $10 \mu \mathrm{M})$ (Sigma, St. Louis, MO, USA) treatment for $1 \mathrm{~h}$ were harvested and lysed in RIPA buffer (Beyotime Institute of Biotechnolgy, Haimen, China) for $30 \mathrm{~min}$. The bicinchoninic acid (BCA) Protein Assay Kit (CoWin Biotechnology, Beijing,
China) was used to detect the concentrations of proteins. Subsequently, samples $(50 \mu \mathrm{g})$ were separated on $10 \%$ sodium dodecylsulfate-polyacrylamide gel electrophoresis (SDS-PAGE) (Beyotime Institute of Biotechnolgy) and then blotted onto PVDF membranes (Millipore, Billerica, MA,USA). Following blocked with $5 \%$ milk in Trisbuffer saline (TBST) at $37^{\circ} \mathrm{C}$ for $1 \mathrm{~h}$, the membranes were incubated with monoclonal antibodies (MDR1/Pgp, p-Raf-1, Raf-1, p-c-Jun (Ser73), c-Jun, c-Fos, and $\beta$-actin) (Santa Cruz Biotechnology, Santa Cruz, CA, USA) with 1:500, 1:1000, 1:500, 1:1000, 1:500, 1:500, and 1:2000 dilutions overnight at $4{ }^{\circ} \mathrm{C}$, respectively. The membranes were then washed three times with TBST and labeled with horseradishperoxidase-conjugated anti-mouse secondary antibodies (1:5000 dilutions). Following washing three times, the blots were visualized by Electrochemiluminescence Plus Detection system (EMD Millipore, Billerica, MA, USA) and analyzed using an Image Scanner (Amersham Biosciences, Uppsala, Sweden). The $\beta$-actin was used as the internal control.

\section{Cell apoptosis assay}

MCF-7/ADR and MCF-7/PTX cells transfected with Gal siRNA1, Gal siRNA2, si-control, pcDNA 3.1MDR1, or pcDNA 3.1 empty vectors were seeded in sixwell plates at a density of $2 \times 10^{5}$ cells/well. Following incubation for $24 \mathrm{~h}$, cells were treated with $15 \mathrm{nM}$ PTX or $300 \mathrm{nM}$ ADR for $48 \mathrm{~h}$. Then the cells were collected and washed three times with PBS, followed by centrifugation at $2 \times 10^{3} \mathrm{rpm}$ for $5 \mathrm{~min}$ and resuspended in $100 \mu \mathrm{L}$ binding buffer. Subsequently, $5 \mu \mathrm{L}$ Annexin V-FITC was added for incubation for $10 \mathrm{~min}$, followed by $5 \mu \mathrm{L}$ propidium iodide (PI) for 15 min incubation. The apoptotic cells were analyzed by using the FACScan flow cytometry and CellQuest software (BD Biosciences, San Jose, CA, USA).

\section{Statistical analysis}

Data from each group were presented as the mean \pm standard deviation (SD) error from at least three independent experiments. Comparison between two groups was performed by Student's $t$ test and differences for three or more groups were analyzed using one-way ANOVA tests. Statistical analysis was conducted using graph prism 5.0 software (GraphPad Prism, San Diego, CA). A statistically significant difference was considered at a $P$ value less than 0.05 .

\section{Abbreviations}

Gal-1, Galectin-1; PTX, paclitaxel; ADR, adriamycin; MDR, multidrug resistance; ATP, adenosine triphosphate; P-gp, P-glycoprotein; MAPK, mitogen-activated protein; Con siRNA, control siRNA; EOC, epithelial ovarian cancer; MMP-9, matrix metalloprotein 9. 


\section{Author contributions}

This work was conceived and designed by Fang Wang and Pengwei Lv. The experiments were carried out by Yuanting Gu and Lin Li. Data were interpreted by Xin Ge. The manuscript was prepared by Guangcheng Guo and Fang Wang.

\section{CONFLICTS OF INTEREST}

All the authors declared no competing interests.

\section{REFERENCES}

1. Dai X, Li T, Bai Z, Yang Y, Liu X, Zhan J, Shi B. Breast cancer intrinsic subtype classification, clinical use and future trends. Am J Cancer Res. 2015; 5:2929-43.

2. van Vlerken LE, Duan Z, Little SR, Seiden MV, Amiji MM. Biodistribution and pharmacokinetic analysis of Paclitaxel and ceramide administered in multifunctional polymerblend nanoparticles in drug resistant breast cancer model. Mol Pharm. 2008; 5:516-26.

3. Vtorushin SV, Khristenko KY, Zavyalova MV, Perelmuter VM, Litviakov NV, Denisov EV, Dulesova AY, Cherdyntseva NV. The phenomenon of multi-drug resistance in the treatment of malignant tumors. Exp Oncol. 2014; 36:144-56.

4. Guo Y, Chu M, Tan S, Zhao S, Liu H, Otieno BO, Yang $\mathrm{X}, \mathrm{Xu} \mathrm{C}$, Zhang Z. Chitosan-g-TPGS nanoparticles for anticancer drug delivery and overcoming multidrug resistance. Mol Pharm. 2014; 11:59-70.

5. Wu Q, Yang Z, Nie Y, Shi Y, Fan D. Multi-drug resistance in cancer chemotherapeutics: mechanisms and lab approaches. Cancer Lett. 2014; 347:159-66.

6. Li D, Zhou L, Huang J, Xiao X. Effect of multidrug resistance 1/P-glycoprotein on the hypoxia-induced multidrug resistance of human laryngeal cancer cells. Oncol Lett. 2016; 12:1569-74.

7. Peer D, Karp JM, Hong S, Farokhzad OC, Margalit R, Langer R. Nanocarriers as an emerging platform for cancer therapy. Nat Nanotechnol. 2007; 2:751-60.

8. Szakács G, Paterson JK, Ludwig JA, Booth-Genthe C, Gottesman MM. Targeting multidrug resistance in cancer. Nat Rev Drug Discov. 2006; 5:219-34.

9. Tang D, Yuan Z, Xue X, Lu Z, Zhang Y, Wang H, Chen M, An Y, Wei J, Zhu Y, Miao Y, Jiang K. High expression of Galectin-1 in pancreatic stellate cells plays a role in the development and maintenance of an immunosuppressive microenvironment in pancreatic cancer. Int J Cancer. 2012; 130:2337-48.

10. Astorgues-Xerri L, Riveiro ME, Tijeras-Raballand A, Serova M, Neuzillet C, Albert S, Raymond E, Faivre S. Unraveling galectin-1 as a novel therapeutic target for cancer. Cancer Treat Rev. 2014; 40:307-19.
11. Upreti M, Jamshidi-Parsian A, Apana S, Berridge M, Fologea DA, Koonce NA, Henry RL, Griffin RJ. Radiationinduced galectin-1 by endothelial cells: a promising molecular target for preferential drug delivery to the tumor vasculature. J Mol Med (Berl). 2013; 91:497-506.

12. Zhong J. RAS and downstream RAF-MEK and PI3KAKT signaling in neuronal development, function and dysfunction. Biol Chem. 2016; 397:215-22.

13. Karreth FA, DeNicola GM, Winter SP, Tuveson DA. C-Raf inhibits MAPK activation and transformation by B-Raf(V600E). Mol Cell. 2009; 36:477-86.

14. Pandey V, Bhaskara VK, Babu PP. Implications of mitogenactivated protein kinase signaling in glioma. J Neurosci Res. 2016; 94:114-27.

15. Blaževitš O, Mideksa YG, Šolman M, Ligabue A, Ariotti N, Nakhaeizadeh H, Fansa EK, Papageorgiou AC, Wittinghofer A, Ahmadian MR, Abankwa D. Galectin-1 dimers can scaffold Raf-effectors to increase H-ras nanoclustering. Sci Rep. 2016; 6:24165.

16. Yu Z, Sato S, Trackman PC, Kirsch KH, Sonenshein GE. Blimp1 activation by AP-1 in human lung cancer cells promotes a migratory phenotype and is inhibited by the lysyl oxidase propeptide. PLoS One. 2012; 7:e33287.

17. Luff SA, Papoutsakis ET. Megakaryocytic Maturation in Response to Shear Flow Is Mediated by the Activator Protein 1 (AP-1) Transcription Factor via Mitogen-activated Protein Kinase (MAPK) Mechanotransduction. J Biol Chem. 2016; 291:7831-43.

18. Papoudou-Bai A, Hatzimichael E, Barbouti A, Kanavaros P. Expression patterns of the activator protein-1 (AP-1) family members in lymphoid neoplasms. Clin Exp Med. 2016. doi: 10.1007/s10238-016-0436-z.

19. Zhu X, Wang K, Zhang K, Xu F, Yin Y, Zhu L, Zhou F. Galectin-1 knockdown in carcinoma-associated fibroblasts inhibits migration and invasion of human MDA-MB-231 breast cancer cells by modulating MMP-9 expression. Acta Biochim Biophys Sin (Shanghai). 2016; 48:462-67.

20. Zhou X, Li D, Wang X, Zhang B, Zhu H, Zhao J. Galectin-1 is overexpressed in $\mathrm{CD} 133+$ human lung adenocarcinoma cells and promotes their growth and invasiveness. Oncotarget. 2015; 6:3111-22. doi: 10.18632/ oncotarget.3076.

21. Jiang K, Wang X, Geng H, Beer TM, Qian DZ, Ramstrom O, Yan M. Carbohydrate-conjugated fluorescent silica nanoprobes for selective detection of galectin-1 and prostate cancer cells. Sci Lett J. 2015; 4.pii: 132.

22. Grosset AA, Labrie M, Vladoiu MC, Yousef EM, Gaboury L, St-Pierre Y. Galectin signatures contribute to the heterogeneity of breast cancer and provide new prognostic information and therapeutic targets. Oncotarget. 2016; 7:18183-203. doi: 10.18632/oncotarget.7784.

23. Astorgues-Xerri L, Riveiro ME, Tijeras-Raballand A, Serova M, Rabinovich GA, Bieche I, Vidaud M, de Gramont A, Martinet M, Cvitkovic E, Faivre S, Raymond 
E. OTX008, a selective small-molecule inhibitor of galectin-1, downregulates cancer cell proliferation, invasion and tumour angiogenesis. Eur J Cancer. 2014; 50:2463-77.

24. Zhang P, Zhang P, Shi B, Zhou M, Jiang H, Zhang H, Pan X, Gao H, Sun H, Li Z. Galectin-1 overexpression promotes progression and chemoresistance to cisplatin in epithelial ovarian cancer. Cell Death Dis. 2014; 5:e991.

25. Zheng L, Xu C, Guan Z, Su X, Xu Z, Cao J, Teng L. Galectin-1 mediates TGF- $\beta$-induced transformation from normal fibroblasts into carcinoma-associated fibroblasts and promotes tumor progression in gastric cancer. Am J Transl Res. 2016; 8:1641-58.

26. Wiest I, Seliger C, Walzel H, Friese K, Jeschke U. Induction of apoptosis in human breast cancer and trophoblast tumor cells by galectin-1. Anticancer Res. $2005 ; 25: 1575-80$.

27. Mi YJ, Liang YJ, Huang HB, Zhao HY, Wu CP, Wang F, Tao LY, Zhang CZ, Dai CL, Tiwari AK, Ma XX, To KK, Ambudkar SV, et al. Apatinib (YN968D1) reverses multidrug resistance by inhibiting the efflux function of multiple ATP-binding cassette transporters. Cancer Res. 2010; 70:7981-91.

28. He Y, Bi Y, Hua Y, Liu D, Wen S, Wang Q, Li M, Zhu J, Lin T, He D, Li X, Wang Z, Wei G. Ultrasound microbubblemediated delivery of the siRNAs targeting MDR1 reduces drug resistance of yolk sac carcinoma L2 cells. J Exp Clin Cancer Res. 2011; 30:104.

29. Liu C, Zhao G, Liu J, Ma N, Chivukula P, Perelman L, Okada K, Chen Z, Gough D, Yu L. Novel biodegradable lipid nano complex for siRNA delivery significantly improving the chemosensitivity of human colon cancer stem cells to paclitaxel. J Control Release. 2009; 140:277-83.

30. Abrams SL, Steelman LS, Shelton JG, Wong EW, Chappell WH, Bäsecke J, Stivala F, Donia M, Nicoletti F, Libra M, Martelli AM, McCubrey JA. The Raf/MEK/ERK pathway can govern drug resistance, apoptosis and sensitivity to targeted therapy. Cell Cycle. 2010; 9:1781-91.

31. Mukherjee R, McGuinness DH, McCall P, Underwood MA, Seywright M, Orange C, Edwards J. Upregulation of MAPK pathway is associated with survival in castrateresistant prostate cancer. Br J Cancer. 2011; 104:1920-28.

32. Kreiseder B, Holper-Schichl YM, Muellauer B, Jacobi N, Pretsch A, Schmid JA, de Martin R, Hundsberger H, Eger A, Wiesner C. Alpha-catulin contributes to drug-resistance

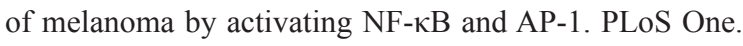
2015; 10:e0119402.

33. Yang G, Wu D, Zhu J, Jiang O, Shi Q, Tian J, Weng Y. Upregulation of miR-195 increases the sensitivity of breast cancer cells to Adriamycin treatment through inhibition of Raf-1. Oncol Rep. 2013; 30:877-89.

34. Chen Q, Bian Y, Zeng S. Involvement of AP-1 and NF-kB in the up-regulation of P-gp in vinblastine resistant Caco-2 cells. Drug Metab Pharmacokinet. 2014; 29:223-26.

35. Bentires-Alj M, Barbu V, Fillet M, Chariot A, Relic B, Jacobs N, Gielen J, Merville MP, Bours V. NF-kappaB transcription factor induces drug resistance through MDR1 expression in cancer cells. Oncogene. 2003; 22:90-97.

36. Luo W, Song L, Chen XL, Zeng XF, Wu JZ, Zhu CR, Huang T, Tan XP, Lin XM, Yang Q, Wang JZ, Li XK, $\mathrm{Wu}$ XP. Identification of galectin-1 as a novel mediator for chemoresistance in chronic myeloid leukemia cells. Oncotarget. 2016; 7:26709-23. doi: 10.18632/ oncotarget.8489.

37. Chen SY, Hu SS, Dong Q, Cai JX, Zhang WP, Sun JY, Wang TT, Xie J, He HR, Xing JF, Lu J, Dong YL. Establishment of paclitaxel-resistant breast cancer cell line and nude mice models, and underlying multidrug resistance mechanisms in vitro and in vivo. Asian Pac J Cancer Prev. 2013; 14:6135-40. 